\title{
On a characterization theorem for the group of $p$-adic numbers Gennadiy Feldman
}

\author{
B.Verkin Institute for Low Temperature Physics and Engineering \\ of the National Academy of Sciences of Ukraine, Kharkov, Ukraine \\ feldman@ilt.kharkov.ua
}

\begin{abstract}
It is well known Heyde's characterization of the Gaussian distribution on the real line: Let $\xi_{1}, \xi_{2}, \ldots, \xi_{n}, n \geq 2$, be independent random variables, let $\alpha_{j}, \beta_{j}$ be nonzero constants such that $\beta_{i} \alpha_{i}^{-1}+\beta_{j} \alpha_{j}^{-1} \neq 0$ for all $i \neq j$. If the conditional distribution of the linear form $L_{2}=\beta_{1} \xi_{1}+\beta_{2} \xi_{2}+\cdots+\beta_{n} \xi_{n}$ given $L_{1}=\alpha_{1} \xi_{1}+\alpha_{2} \xi_{2}+\cdots+\alpha_{n} \xi_{n}$ is symmetric, then all random variables $\xi_{j}$ are Gaussian. We prove an analogue of this theorem for two independent random variables in the case when they take values in the group of $p$-adic numbers $\Omega_{p}$, and coefficients of linear forms are topological automorphisms of $\Omega_{p}$.
\end{abstract}

Keywords Linear forms, conditional distribution, group of $p$-adic numbers

Mathematics Subject Classification 60B15, 62E10, 43A35

\section{Introduction}

It is well known Heyde's characterization of the Gaussian distribution on the real line by the symmetry of the conditional distribution of one linear form given another ([8], see also [9, $\S 13.4 .1])$ :

Theorem A. Let $\xi_{1}, \xi_{2}, \ldots, \xi_{n}, n \geq 2$, be independent random variables, let $\alpha_{j}, \beta_{j}$ be nonzero constants such that $\beta_{i} \alpha_{i}^{-1}+\beta_{j} \alpha_{j}^{-1} \neq 0$ for all $i \neq j$. If the conditional distribution of the linear form $L_{2}=\beta_{1} \xi_{1}+\beta_{2} \xi_{2}+\cdots+\beta_{n} \xi_{n}$ given $L_{1}=\alpha_{1} \xi_{1}+\alpha_{2} \xi_{2}+\cdots+\alpha_{n} \xi_{n}$ is symmetric, then all random variables $\xi_{j}$ are Gaussian.

Let $X$ be a second countable locally compact Abelian group, $\operatorname{Aut}(X)$ be the group of topological automorphisms of $X, \xi_{j}, j=1,2, \ldots, n, n \geq 2$, be independent random variables with values in $X$ and distributions $\mu_{j}$. Consider the linear forms $L_{1}=\alpha_{1} \xi_{1}+\alpha_{2} \xi_{2}+\cdots+\alpha_{n} \xi_{n}$ and $L_{2}=\beta_{1} \xi_{1}+\beta_{2} \xi_{2}+\cdots+\beta_{n} \xi_{n}$, where $\alpha_{j}, \beta_{j} \in \operatorname{Aut}(X)$. We formulate the general problem.

Problem 1. Let $X$ be a given group. Describe distributions $\mu_{j}$ assuming that the conditional distribution of $L_{2}$ given $L_{1}$ is symmetric. In particular, for which groups $X$ the symmetry of the conditional distribution of $L_{2}$ given $L_{1}$ implies that all $\mu_{j}$ are either Gaussian distributions or belong to a class of distributions which we can consider as a natural analogue of the class of Gaussian distributions.

This problem for different classes of locally compact Abelian groups was studied in series articles (see [1]- 3], [5], 6], [10]-[12]). In this article we continue these investigations. We study Problem 1 for two independent random variables with values in the group of $p$-adic numbers.

We will use some results of the duality theory for locally compact Abelian groups (see e.g. [7]). Before we formulate the main theorem remind some definitions and agree on notation. For an arbitrary locally compact Abelian group $X$ let $Y=X^{*}$ be its character group, and $(x, y)$ be the value of a character $y \in Y$ at an element $x \in X$. If $K$ is a closed subgroup of 
$X$, we denote by $A(Y, K)=\{y \in Y:(x, y)=1$ for all $x \in K\}$ its annihilator. If $\delta: X \mapsto X$ is a continuous endomorphism, then the adjoint endomorphism $\widetilde{\delta}: Y \mapsto Y$ is defined by the formula $(x, \widetilde{\delta} y)=(\delta x, y)$ for all $x \in X, y \in Y$. We note that $\delta \in \operatorname{Aut}(X)$ if and only if $\widetilde{\delta} \in \operatorname{Aut}(Y)$. Denote by $I$ the identity automorphism of a group.

Let $M^{1}(X)$ be the convolution semigroup of probability distributions on $X$. For a distribution $\mu \in M^{1}(X)$ denote by $\widehat{\mu}(y)=\int_{X}(x, y) d \mu(x)$ its characteristic function. If $H$ is a closed subgroup of $Y$ and $\widehat{\mu}(y)=1$ for $y \in H$, then $\widehat{\mu}(y+h)=\widehat{\mu}(y)$ for all $y \in Y, h \in H$. For $\mu \in M^{1}(X)$, we define the distribution $\bar{\mu} \in M^{1}(X)$ by the formula $\bar{\mu}(E)=\mu(-E)$ for any Borel set $E \subset X$. Observe that $\widehat{\bar{\mu}}(y)=\overline{\widehat{\mu}(y)}$. Let $K$ be a compact subgroup of $X$. Note that the characteristic function of the Haar distribution $m_{K}$ is of the form

$$
\widehat{m}_{K}(y)= \begin{cases}1, & y \in A(Y, K) \\ 0, & y \notin A(Y, K)\end{cases}
$$

Denote by $I(X)$ the set of the idempotent distributions on $X$, i.e. the set of shifts of the Haar distributions $m_{K}$ of compact subgroups $K$ of $X$.

\section{The main theorem}

Let $p$ be a prime number. We need some properties of the group of $p$-adic numbers $\Omega_{p}$ (see e.g. [7, §10]). As a set $\Omega_{p}$ coincides with the set of sequences of integers of the form $x=\left(\ldots, x_{-n}, x_{-n+1}, \ldots, x_{-1}, x_{0}, x_{1}, \ldots, x_{n}, \ldots\right)$, where $x_{n} \in\{0,1, \ldots, p-1\}$, such that $x_{n}=0$ for $n<n_{0}$, where the number $n_{0}$ depends on $x$. Correspond to each element $x \in \Omega_{p}$ the series $\sum_{k=-\infty}^{\infty} x_{k} p^{k}$. Addition and multiplication of series are defined in a natural way and define the operations of addition and multiplication in $\Omega_{p}$. With respect to these operations $\Omega_{p}$ is a field. Denote by $\Delta_{p}$ a subgroup of $\Omega_{p}$ consisting of $x \in \Omega_{p}$ such that $x_{n}=0$ for $n<0$. This subgroup is called the group of $p$-adic integers. Elements of the group $\Delta_{p}$ we write in the form $x=\left(x_{0}, x_{1}, \ldots, x_{n}, \ldots\right)$. The family of subgroups $\left\{p^{m} \Delta_{p}\right\}_{m=-\infty}^{\infty}$ can be considered as an open basis at zero of the group $\Omega_{p}$ and defines a topology on $\Omega_{p}$. With respect to this topology the group $\Omega_{p}$ is locally compact, noncompact, and totally disconnected. The character group $\Omega_{p}^{*}$ of the group $\Omega_{p}$ is topologically isomorphic to $\Omega_{p}$, and the value of a character $y \in \Omega_{p}^{*}$ at an element $x \in \Omega_{p}$ is defined by the formula

$$
(x, y)=\exp \left[2 \pi i\left(\sum_{n=-\infty}^{\infty} x_{n}\left(\sum_{s=n}^{\infty} y_{-s} p^{-s+n-1}\right)\right)\right]
$$

([7. $(25.1)])$.

Each automorphism $\alpha \in \operatorname{Aut}\left(\Omega_{p}\right)$ is the multiplication by an element $x_{\alpha} \in \Omega_{p}, x_{\alpha} \neq 0$, i.e. $\alpha g=x_{\alpha} g, g \in \Omega_{p}$. If $\alpha \in \operatorname{Aut}\left(\Omega_{p}\right)$, in order not to complicate notation, we will identify the automorphism $\alpha$ with the corresponding element $x_{\alpha}$, i.e. when we write $\alpha g$, we will suppose that $\alpha \in \Omega_{p}$. We note that $\widetilde{\alpha}=\alpha$. Denote by $\Delta_{p}^{0}$ the subset of $\Delta_{p}$, consisting of all invertible elements of $\Delta_{p}, \Delta_{p}^{0}=\left\{x=\left(x_{0}, x_{1}, \ldots, x_{n}, \ldots\right) \in \Delta_{p}: x_{0} \neq 0\right\}$. Obviously, each element $g \in \Omega_{p}$ is represented in the form $g=p^{k} c$, where $k$ is an integer, and $c \in \Delta_{p}^{0}$. It is obvious that the multiplication by $c$ is a topological automorphism of the group $p^{m} \Delta_{p}$ for any integer $m$.

For a fixed prime $p$ denote by $\mathbf{Z}\left(p^{\infty}\right)$ the set of rational numbers of the form

$$
\left\{\frac{k}{p^{n}}: k=0,1, \ldots, p^{n}-1, n=0,1, \ldots\right\}
$$


and define the operation in $\mathbf{Z}\left(p^{\infty}\right)$ as addition modulo 1 . Then $\mathbf{Z}\left(p^{\infty}\right)$ is transformed into an Abelian group, which we consider in the discrete topology. Obviously, this group is topologically isomorphic to the multiplicative group of $p^{n}$ th roots of unity, where $n$ goes through the nonnegative integers, considering in the discrete topology. For a fixed $n$ denote by $\mathbf{Z}\left(p^{n}\right)$ the subgroup of $\mathbf{Z}\left(p^{\infty}\right)$ consisting of all elements of the form $\left\{\frac{k}{p^{n}}: k=0,1, \ldots, p^{n}-1\right\}$. The group $\mathbf{Z}\left(p^{n}\right)$ is isomorphic to the multiplicative group of $p^{n}$ th roots of unity.

Let $\alpha \in \operatorname{Aut}\left(\Omega_{p}\right), \alpha=p^{k} c$, where $k \geq 0, c \in \Delta_{p}^{0}$. Let $l$ be an integer. It is easy to see that $\alpha$ induces an epimorphism $\bar{\alpha}$ and $c$ induces an automorphism $\bar{c}$ on the factor-group $\Omega_{p} / p^{l} \Delta_{p}$. Let

$$
x=\left(\ldots, x_{-n}, x_{-n+1}, \ldots, x_{-1}, x_{0}, x_{1}, \ldots, x_{n}, \ldots\right) \in \Omega_{p} .
$$

Define the mapping $\tau: \Omega_{p} / p^{l} \Delta_{p} \mapsto \mathbf{Z}\left(p^{\infty}\right)$ by the formula

$$
\tau\left(x+p^{l} \Delta_{p}\right)=\sum_{n=-\infty}^{l-1} x_{n} p^{n-l}, \quad x+p^{l} \Delta_{p} \in \Omega_{p} / p^{l} \Delta_{p} .
$$

Then $\tau$ is a topological isomorphism of the groups $\Omega_{p} / p^{l} \Delta_{p}$ and $\mathbf{Z}\left(p^{\infty}\right)$. Put

$$
\widehat{\alpha}=\tau \bar{\alpha} \tau^{-1}, \quad \widehat{c}=\tau \bar{c} \tau^{-1},
$$

and observe that $\widehat{\alpha}=p^{k} \widehat{c}, \widehat{c} \in \operatorname{Aut}\left(\mathbf{Z}\left(p^{\infty}\right)\right)$, and $\widehat{\alpha}$ is an epimorphism. If $c=$ $\left(c_{0}, c_{1}, \ldots, c_{n}, \ldots\right) \in \Delta_{p}^{0}$, then the automorphism $\widehat{c}$ acts in the following way. Put $s_{n}=$ $c_{0}+c_{1} p+c_{2} p^{2}+\ldots+c_{n-1} p^{n-1}$. The restriction of the automorphism $\widehat{c}$ to the subgroup $\mathbf{Z}\left(p^{n}\right) \subset \mathbf{Z}\left(p^{\infty}\right)$ is of the form $\widehat{c} y=s_{n} y, y \in \mathbf{Z}\left(p^{n}\right)$, i.e. $\widehat{c}$ acts in $\mathbf{Z}\left(p^{n}\right)$ as the multiplication by $s_{n}$.

We note that since $\Omega_{p}$ is a totally disconnected group, the Gaussian distributions on $\Omega_{p}$ are degenerated ([13, Ch. 4]), and the class of idempotent distributions on $\Omega_{p}$ can be considered as a natural analog of the class of Gaussian distributions.

We consider the case of two independent random variables $\xi_{1}$ and $\xi_{2}$ with values in the group $X=\Omega_{p}$, i.e. we consider the linear forms $L_{1}=\alpha_{1} \xi_{1}+\alpha_{2} \xi_{2}$ and $L_{2}=\beta_{2} \xi_{1}+\beta_{2} \xi_{2}$, where $\alpha_{j}, \beta_{j} \in \operatorname{Aut}(X)$. Assume that the conditional distribution of the linear form $L_{2}=\beta_{2} \xi_{1}+\beta_{2} \xi_{2}$ given $L_{1}=\alpha_{1} \xi_{1}+\alpha_{2} \xi_{2}$ is symmetric. Our aim is to describe the possible distributions $\mu_{j}$ depending on $\alpha_{j}, \beta_{j}$. Introducing the new independent random variables $\xi_{j}^{\prime}=\alpha_{j} \xi_{j}, j=1,2$, we can suppose that $L_{1}=\xi_{1}+\xi_{2}$ and $L_{2}=\delta_{1} \xi_{1}+\delta_{2} \xi_{2}$, where $\delta_{j} \in \operatorname{Aut}(X)$. Note also that the conditional distribution of the linear form $L_{2}=\delta_{1} \xi_{1}+\delta_{2} \xi_{2}$ given $L_{1}=\xi_{1}+\xi_{2}$ is symmetric if and only if the conditional distribution of $\delta L_{2}$, where $\delta \in \operatorname{Aut}(X)$, given $L_{1}$ is symmetric. It follows from this that we may assume that $L_{2}=\xi_{1}+\alpha \xi_{2}$, where $\alpha \in \operatorname{Aut}(X)$.

We can formulate now the main result of the article.

Theorem 1. Let $X=\Omega_{p}$. Let $\alpha=p^{k} c$, where $k \in \mathbf{Z}$ and $c=\left(c_{0}, c_{1}, \ldots, c_{n}, \ldots\right) \in \Delta_{p}^{0}$, be an arbitrary topological automorphism of the group $X$. Then the following statements hold.

1. Let $\xi_{1}$ and $\xi_{2}$ be independent random variables with values in $X$ and distributions $\mu_{1}$ and $\mu_{2}$. Assume that the conditional distribution of the linear form $L_{2}=\xi_{1}+\alpha \xi_{2}$ given $L_{1}=\xi_{1}+\xi_{2}$ is symmetric. Then

$1(i)$ If $p>2, k=0$ and $c_{0} \neq p-1$, then $\mu_{j}=m_{K} * E_{x_{j}}$, where $K$ is a compact subgroup of $X$ and $x_{j} \in X$. Moreover, if $c_{0}=1$, then $\mu_{1}$ and $\mu_{2}$ are degenerate distributions.

$1($ ii $)$ If $p=2, k=0, c_{0}=1$ and $c_{1}=0$, then $\mu_{1}$ and $\mu_{2}$ are degenerate distributions.

$1($ iii $)$ If $p>2$ and $|k|=1$, then either $\mu_{1} \in I(X)$ or $\mu_{2} \in I(X)$. 
2. If one of the following conditions holds:

2 (i) $p>2, k=0, c_{0}=p-1$

2 (ii) $p=2, k=0, c_{0}=c_{1}=1$;

$2($ iii) $p=2,|k|=1$;

$2(i v) p \geq 2,|k| \geq 2$,

then there exist independent random variables $\xi_{1}$ and $\xi_{2}$ with values in $X$ and distributions $\mu_{1}$ and $\mu_{2}$ such that the conditional distribution of the linear form $L_{2}=\xi_{1}+\alpha \xi_{2}$ given $L_{1}=\xi_{1}+\xi_{2}$ is symmetric whereas $\mu_{1}, \mu_{2} \notin I(X)$.

It is easy to see that cases $1(i)-1(i i i)$ and $2(i)-2(i v)$ exhaust all possibilities for $p$ and $\alpha$.

\section{Lemmas}

To prove Theorem 1 we need some lemmas.

Lemma 1 ([3], see also [4, §16]). Let $X$ be a second countable locally compact Abelian group, $Y$ be its character group. Let $\xi_{1}$ and $\xi_{2}$ be independent random variables with values in $X$ and distributions $\mu_{1}$ and $\mu_{2}$. Let $\alpha_{j}, \beta_{j}$ be continuous endomorphisms of $X$. The conditional distribution of the linear form $L_{2}=\beta_{1} \xi_{1}+\beta_{2} \xi_{2}$ given $L_{1}=\alpha_{1} \xi_{1}+\alpha_{2} \xi_{2}$ is symmetric if and only if the characteristic functions $\widehat{\mu}_{j}(y)$ satisfy the equation

$$
\widehat{\mu}_{1}\left(\widetilde{\alpha}_{1} u+\widetilde{\beta}_{1} v\right) \widehat{\mu}_{2}\left(\widetilde{\alpha}_{2} u+\widetilde{\beta}_{2} v\right)=\widehat{\mu}_{1}\left(\widetilde{\alpha}_{1} u-\widetilde{\beta}_{1} v\right) \widehat{\mu}_{2}\left(\widetilde{\alpha}_{2} u-\widetilde{\beta}_{2} v\right), \quad u, v \in Y .
$$

Observe that in [3] this lemma is proved when $\alpha_{j}, \beta_{j} \in \operatorname{Aut}(X)$, but the proof is valid without any changes for the case when $\alpha_{j}, \beta_{j}$ are continuous endomorphisms.

Lemma 2. Let $X=\Omega_{p}, \xi_{1}$ and $\xi_{2}$ be independent random variables with values in $X$ and distributions $\mu_{1}$ and $\mu_{2}$ such that $\mu_{j}(y) \geq 0, j=1,2$. Let $\alpha=p^{k} c \in \operatorname{Aut}(X)$, where $k \in \mathbf{Z}$ and $c \in \Delta_{p}^{0}$. Assume also that $\alpha \neq-I$. If the conditional distribution of the linear form $L_{2}=\xi_{1}+\alpha \xi_{2}$ given $L_{1}=\xi_{1}+\xi_{2}$ is symmetric, then there exists a closed subgroup $H \subset Y$, such that $\widehat{\mu}_{j}(y)=1$ for $y \in H, j=1,2$.

Proof. Taking into account that the conditional distribution of the linear form $L_{2}$ given $L_{1}$ is symmetric if and only if the conditional distribution of the linear form $\alpha^{-1} L_{2}$ given $L_{1}$ is symmetric, we may assume without loss of generality that $k \geq 0$. Since $X=\Omega_{p}$, we have $\widetilde{\alpha}=\alpha$, and hence equation (4) takes the form

$$
\widehat{\mu}_{1}(u+v) \widehat{\mu}_{2}(u+\alpha v)=\widehat{\mu}_{1}(u-v) \widehat{\mu}_{2}(u-\alpha v), \quad u, v \in Y .
$$

If $\alpha=I$, then put in equation (5) $u=v=y$. We get that $H=Y$, i.e. $\mu_{1}$, and $\mu_{2}$ are degenerate distributions. So, we will assume that $\alpha \neq I$. Since $\widehat{\mu}_{1}(0)=\widehat{\mu}_{2}(0)=1$, we can choose a neighborhood at zero $V$ of $Y$ such that $\widehat{\mu}_{j}(y)>0$ for $y \in V, j=1,2$. Obviously, we can assume that $V=p^{l} \Delta_{p}$ for some $l$. Since $k \geq 0$, we have $\alpha\left(p^{l} \Delta_{p}\right) \subset p^{l} \Delta_{p}$. Put $\psi_{j}(y)=-\log \widehat{\mu}_{j}(y), y \in p^{l} \Delta_{p}, j=1,2$. It follows from (5) that the functions $\psi_{1}(y)$ and $\psi_{2}(y)$ satisfy the equation

$$
\psi_{1}(u+v)+\psi_{2}(u+\alpha v)-\psi_{1}(u-v)-\psi_{2}(u-\alpha v)=0, \quad u, v \in p^{l} \Delta_{p} .
$$

We use the finite difference method to solve equation (6). Let $\psi(y)$ be a function on $Y$, and $h$ be an arbitrary element of $Y$. Denote by $\Delta_{h}$ the finite difference operator

$$
\Delta_{h} \psi(y)=\psi(y+h)-\psi(y) .
$$


Let $k_{1}$ be an arbitrary element of $p^{l} \Delta_{p}$. Put $h_{1}=\alpha k_{1}$ and hence, $h_{1}-\alpha k_{1}=0$. Substitute $u+h_{1}$ for $u$ and $v+k_{1}$ for $v$ in equation (6) Subtracting equation (6) from the obtained equation we find

$$
\Delta_{l_{11}} \psi_{1}(u+v)+\Delta_{l_{12}} \psi_{2}(u+\alpha v)-\Delta_{l_{13}} \psi_{1}(u-v)=0, \quad u, v \in p^{l} \Delta_{p},
$$

where $l_{11}=(\alpha+I) k_{1}, l_{12}=2 \alpha k_{1}, l_{13}=(\alpha-I) k_{1}$. Let $k_{2}$ be an arbitrary element of $p^{l} \Delta_{p}$. Put $h_{2}=k_{2}$ and hence, $h_{2}-k_{2}=0$. Substitute $u+h_{2}$ for $u$ and $v+k_{2}$ for $v$ in equation (7). Subtracting equation (7) from the obtained equation we arrive at

$$
\Delta_{l_{21}} \Delta_{l_{11}} \psi_{1}(u+v)+\Delta_{l_{22}} \Delta_{l_{12}} \psi_{2}(u+\alpha v)=0, \quad u, v \in p^{l} \Delta_{p},
$$

where $l_{21}=2 k_{2}, l_{22}=(I+\alpha) k_{2}$. Let $k_{3}$ be an arbitrary element of $p^{l} \Delta_{p}$. Put $h_{3}=-\alpha k_{3}$ and hence, $h_{3}+\alpha k_{3}=0$. Substitute $u+h_{3}$ for $u$ and $v+k_{3}$ for $v$ in equation (8) . Subtracting equation (8) from the obtained equation we find

$$
\Delta_{l_{31}} \Delta_{l_{21}} \Delta_{l_{11}} \psi_{1}(u+v)=0, \quad u, v \in p^{l} \Delta_{p}
$$

where $l_{31}=(I-\alpha) k_{3}$. Substituting $v=0$ into (9), taking into account the expressions for $l_{11}, l_{21}, l_{31}$ and the fact that $k_{1}, k_{2}, k_{3}$ are arbitrary elements of $p^{l} \Delta_{p}$, we find from (9) that there exists a subgroup $p^{m} \Delta_{p} \subset p^{l} \Delta_{p}$, where the function $\psi_{1}(y)$ satisfies the equation

$$
\Delta_{h}^{3} \psi_{1}(y)=0, \quad h, y \in p^{m} \Delta_{p} .
$$

Taking into account that $p^{m} \Delta_{p}$ is a compact group, we conclude from (10) that $\psi_{1}(y)=$ const, $y \in p^{m} \Delta_{p}([4, \S 5])$. Since $\psi_{1}(0)=0$, we have $\psi_{1}(y)=0$, for $y \in p^{m} \Delta_{p}$. This implies that $\widehat{\mu}_{1}(y)=1$ for $y \in p^{m} \Delta_{p}$. For the distribution $\mu_{2}$ we reason similarly and find a subgroup $p^{n} \Delta_{p}$ such that $\widehat{\mu}_{2}(y)=1$ for $y \in p^{n} \Delta_{p}$. Put $H=p^{m} \Delta_{p} \cap p^{n} \Delta_{p}$. Lemma 2 is proved.

Lemma 3 ([1], see also [4, $\$ 16])$. Let $X$ be a finite Abelian group containing no elements of order 2, $\alpha$ be an automorphism of $X$ such that $I \pm \alpha \in \operatorname{Aut}(X)$. Let $\xi_{1}$ and $\xi_{2}$ be independent random variables with values in the group $X$ and distributions $\mu_{1}$ and $\mu_{2}$. If the conditional distribution of the linear form $L_{2}=\xi_{1}+\alpha \xi_{2}$ given $L_{1}=\xi_{1}+\xi_{2}$ is symmetric, then $\mu_{j}=m_{K} * E_{x_{j}}$, where $K$ is a finite subgroup of $X$ and $x_{j} \in X, j=1,2$.

\section{Proof of statements $1(i)-1(i i i)$}

Before we begin the proof make the following remarks. It is obvious that the characteristic functions of the distributions $\bar{\mu}_{j}$ also satisfy equation (5). This implies that the characteristic functions of the distributions $\nu_{j}=\mu_{j} * \bar{\mu}_{j}$ satisfy equation (5) too. We have $\widehat{\nu}_{j}(y)=\left|\widehat{\mu}_{j}(y)\right|^{2} \geq$ $0, j=1,2$. Hence, when we prove statements $1(i)-1(i i i)$ we may assume without loss of generality that $\mu_{j}(y) \geq 0, j=1,2$, because $\mu_{j}$ and $\nu_{j}$ are idempotent distributions or degenerate distributions simultaneously.

Taking into account that the conditional distribution of the linear form $L_{2}$ given $L_{1}$ is symmetric if and only if the conditional distribution of the linear form $\alpha^{-1} L_{2}$ given $L_{1}$ is symmetric, we may assume without loss of generality that $k \geq 0$.

Put $f(y)=\widehat{\mu}_{1}(y), g(y)=\widehat{\mu}_{2}(y)$ and write equation (5) in the form

$$
f(u+v) g(u+\alpha v)=f(u-v) g(u-\alpha v), \quad u, v \in Y,
$$

where $\alpha \in \operatorname{Aut}(Y), \alpha=p^{k} c, k \geq 0, c \in \Delta_{p}^{0}$. We also assume that $f(y) \geq 0, g(y) \geq 0$. In fact we will study solutions of equation (11). 
Proof of statements $1(i)$ and $1(i i)$. By Lemma 1, the characteristic functions $f(y)$ and $g(y)$ satisfy equation (11). Put

$$
E=\{y \in Y: f(y)=g(y)=1\} .
$$

If either $\mu_{1}$ or $\mu_{1}$ is a nondegenerate distribution, then $E \neq \Omega_{p}$. Observe that in cases $1(i)$ and $1(i i) \alpha \neq-I$. Then by Lemma 2, $E \neq\{0\}$. Thus, $E$ is a nonzero proper closed subgroup of $\Omega_{p}$, and hence $E=p^{l} \Delta_{p}$ for some $l$. It follows from (12) that

$$
f(y+h)=f(y), \quad g(y+h)=g(y), \quad y \in Y, h \in p^{l} \Delta_{p} .
$$

Taking into account (13), denote by $\bar{f}(y)$ and $\bar{g}(y)$ the functions induced by the functions $f(y)$ and $g(y)$ on the factor-group $Y / p^{l} \Delta_{p}$. Put $\hat{f}=\bar{f} \circ \tau^{-1}$, where $\tau$ is defined by formula (2). We get from (11) that the functions $\widehat{f}(y)$ and $\widehat{g}(y)$ satisfy the equation

$$
\widehat{f}(u+v) \widehat{g}(u+\widehat{\alpha} v)=\widehat{f}(u-v) \widehat{g}(u-\widehat{\alpha} v), \quad u, v \in \mathbf{Z}\left(p^{\infty}\right),
$$

where $\widehat{\alpha}$ is defined by formula (3). It follows from (12) that

$$
\left\{y \in \mathbf{Z}\left(p^{\infty}\right): \widehat{f}(y)=\widehat{g}(y)=1\right\}=\{0\} .
$$

Statement $1(i)$. Assume first that $c_{0} \neq 1$. Since $p>2, k=0$ and $c_{0} \neq p-1$, we have

$$
I \pm \widehat{\alpha} \in \operatorname{Aut}\left(\mathbf{Z}\left(p^{\infty}\right)\right)
$$

We note that for any $n$ the restriction of any automorphism of the group $\mathbf{Z}\left(p^{\infty}\right)$ to the subgroup $\mathbf{Z}\left(p^{n}\right) \subset \mathbf{Z}\left(p^{\infty}\right)$ is an automorphism of the subgroup $\mathbf{Z}\left(p^{n}\right)$. Consider the restriction of equation (14) to the subgroup $\mathbf{Z}\left(p^{n}\right)$. Observe that $\left(\mathbf{Z}\left(p^{n}\right)\right)^{*} \cong \mathbf{Z}\left(p^{n}\right)$, and the group $\mathbf{Z}\left(p^{n}\right)$ contains no elements of order 2 . Taking into account that (16) holds, we can apply Lemmas 1 and 3 to the group $\mathbf{Z}\left(p^{n}\right)$ and get that the restrictions of the characteristic functions $\widehat{f}(y)$ and $\widehat{g}(y)$ to the subgroup $\mathbf{Z}\left(p^{n}\right)$ take only two values 0 and 1 . Moreover, $\widehat{f}(y)=\widehat{g}(y)$ for $y \in \mathbf{Z}\left(p^{n}\right)$. Hence, the characteristic functions $\widehat{f}(y)$ and $\widehat{g}(y)$ on the group $\mathbf{Z}\left(p^{\infty}\right)$ take also only two values 0 and 1 , and $\widehat{f}(y)=\widehat{g}(y)$ for $y \in \mathbf{Z}\left(p^{\infty}\right)$. Then the standard reasoning show that $\mu_{j}=m_{K} * E_{x_{j}}$, where $K$ is a compact subgroup of $X$ and $x_{j} \in X$.

Assume now that $c_{0}=1$. Since $k=0$, the restriction of the automorphism $\widehat{\alpha}$ to $\mathbf{Z}(p) \subset$ $\mathbf{Z}\left(p^{\infty}\right)$ is the identity automorphism. Hence, the restriction of equation (14) to $\mathbf{Z}(p)$ takes the form

$$
\widehat{f}(u+v) \widehat{g}(u+v)=\widehat{f}(u-v) \widehat{g}(u-v), \quad u, v \in \mathbf{Z}(p) .
$$

Substituting here $u=v=y$, we get $\widehat{f}(2 y) \widehat{g}(2 y)=1, y \in \mathbf{Z}(p)$. Since $p>2$, this implies that

$$
\widehat{f}(y)=\widehat{g}(y)=1, \quad y \in \mathbf{Z}(p),
$$

but this contradicts to (15). Thus, $\mu_{1}$ and $\mu_{2}$ are degenerate distributions.

Statement $1(i i)$. Since $k=0, c_{0}=1$ and $c_{1}=0$, the restriction of the automorphism $\widehat{\alpha}$ to the subgroup $\mathbf{Z}(4) \subset \mathbf{Z}\left(2^{\infty}\right)$ is the identity automorphism. Hence, the restriction of equation (14) to the subgroup $\mathbf{Z}(4)$ takes the form (17), where $u, v \in \mathbf{Z}(4)$. Substituting in (17) $u=v=y$, we get

$$
\widehat{f}(2 y) \widehat{g}(2 y)=1, \quad y \in \mathbf{Z}(4) .
$$


This implies that $\widehat{f}(y)=\widehat{g}(y)=1$ for $y \in \mathbf{Z}(2)$, but this contradicts to (15). Thus, $\mu_{1}$ and $\mu_{2}$ are degenerate distributions.

The proof of statement $1($ iii $)$ is based on the proof of the following proposition which is of interest in its own right.

Proposition 1. Let $X=\Omega_{p}, \xi_{1}$ and $\xi_{2}$ be independent random variables with values in $X$ and distributions $\mu_{1}$ and $\mu_{2}$. Let $\alpha \in \operatorname{Aut}(X)$, and $\alpha \neq-I$. If the conditional distribution of the linear form $L_{2}=\xi_{1}+\alpha \xi_{2}$ given $L_{1}=\xi_{1}+\xi_{2}$ is symmetric, then either $\mu_{1}$ and $\mu_{2}$ are degenerate distributions or there exists a closed subgroup $M \subset Y$, such that $\widehat{\mu}_{j}(y)=0$ for $y \notin M, j=1,2$.

Proof. Let $\alpha=p^{k} c \in \operatorname{Aut}(X)$, where $k \in \mathbf{Z}$ and $c \in \Delta_{p}^{0}$. We can assume without loss of generality that $k \geq 0$. Otherwise we consider the linear form $L_{2}=\alpha^{-1} \xi_{1}+\xi_{2}$ instead of $L_{2}=\xi_{1}+\alpha \xi_{2}$. Taking into account that in cases $1(i)$ and $1(i i)$ of Theorem 1 Proposition 1 holds, it remains to consider the following cases:

1. $p \geq 2, k \geq 1$;

2. $p>2, k=0, c_{0}=p-1$;

3. $p=2, k=0, c_{0}=c_{1}=1$.

Obviously, we can assume without loss of generality that $\widehat{\mu}_{j}(y) \geq 0, j=1,2$. Put $f(y)=\widehat{\mu}_{1}(y), g(y)=\widehat{\mu}_{2}(y)$. We have $f(-y)=f(y)$ and $g(-y)=g(y)$. Reasoning as in the proof of statements $1(i)-1(i i)$ and retaining the same notation we arrive at equation (14). Set $\beta=I-\alpha, \gamma=I+\alpha$. Substituting into equation (14) $u=v=y$, we obtain

$$
\widehat{f}(2 y) \widehat{g}(\widehat{\gamma} y)=\widehat{g}(\widehat{\beta} y), \quad y \in \mathbf{Z}\left(p^{\infty}\right) .
$$

Substituting into equation (14) $u=\widehat{\alpha} y, v=y$, we get

$$
\widehat{f}(\widehat{\gamma} y) \widehat{g}(2 \widehat{\alpha} y)=\widehat{f}(\widehat{\beta} y), \quad y \in \mathbf{Z}\left(p^{\infty}\right) .
$$

1. $p \geq 2, k \geq 1$. Since $k \geq 1$, we have $\widehat{\beta}, \widehat{\gamma} \in \operatorname{Aut}\left(\mathbf{Z}\left(p^{\infty}\right)\right)$. Put $\kappa=\beta \gamma^{-1}$. Equations (18) and (19) imply

$$
\widehat{g}(\widehat{\kappa} y)=\widehat{f}\left(2 \widehat{\gamma}^{-1} y\right) \widehat{g}(y), \quad y \in \mathbf{Z}\left(p^{\infty}\right)
$$

and

$$
\widehat{f}(\widehat{\kappa} y)=\widehat{f}(y) \widehat{g}\left(2 \widehat{\alpha} \widehat{\gamma}^{-1} y\right), \quad y \in \mathbf{Z}\left(p^{\infty}\right) .
$$

Since $0 \leq \widehat{f}(y) \leq 1$, it follows from (20) that

$$
\widehat{g}(\widehat{\kappa} y) \leq \widehat{g}(y), \quad y \in \mathbf{Z}\left(p^{\infty}\right) .
$$

This implies that for any natural $n$ the inequalities

$$
\widehat{g}\left(\widehat{\kappa}^{n} y\right) \leq \ldots \leq \widehat{g}(\widehat{\kappa} y) \leq \widehat{g}(y), \quad y \in \mathbf{Z}\left(p^{\infty}\right),
$$

hold. Let $y \in \mathbf{Z}\left(p^{\infty}\right)$. Then $y \in \mathbf{Z}\left(p^{l}\right)$ for some $l$, and hence $\widehat{\kappa} y \in \mathbf{Z}\left(p^{l}\right)$. It follows from this that $\widehat{\kappa}^{m} y=y$ for some $m$, depending generally speaking on $y$. Substituting in (22) $n=m$ we get

$$
\widehat{g}(\widehat{\kappa} y)=\widehat{g}(y), \quad y \in \mathbf{Z}\left(p^{\infty}\right) .
$$

Reasoning similarly, we obtain from (21) that

$$
\widehat{f}(\widehat{\kappa} y)=\widehat{f}(y), \quad y \in \mathbf{Z}\left(p^{\infty}\right) .
$$


Assume that there exists a sequence of elements $y_{n} \in \mathbf{Z}\left(p^{\infty}\right)$ such that:

(a) the order of element $y_{n}$ is equal to $p^{i_{n}}, i_{n} \rightarrow \infty$;

(b) $\widehat{g}\left(y_{n}\right) \neq 0$.

Then it follows from (20) and (23) that,

$$
\widehat{f}\left(2 \widehat{\gamma}^{-1} y_{n}\right)=1 \text {. }
$$

Suppose that $p>2$. Then (25) implies that $\widehat{f}(y)=1$ for $y \in \mathbf{Z}\left(p^{i_{n}}\right)$, because the order of the element $2 \widehat{\gamma}^{-1} y_{n}$ is equal to $p^{i_{n}}$, and hence the element $2 \widehat{\gamma}^{-1} y_{n}$ generates the subgroup $\mathbf{Z}\left(p^{i_{n}}\right)$. If $p=2$, then $\widehat{f}(y)=1$ for $y \in \mathbf{Z}\left(2^{i_{n}-1}\right)$, because the order of the element $2 \widehat{\gamma}^{-1} y_{n}$ is equal to $2^{i_{n}-1}$, and the element $2 \widehat{\gamma}^{-1} y_{n}$ generates the subgroup $\mathbf{Z}\left(2^{i_{n}-1}\right)$. Thus, for $p \geq 2$ we have $\widehat{f}(y)=1$ for $y \in \mathbf{Z}\left(p^{\infty}\right)$, and equation (14) implies that

$$
\widehat{g}(u+\widehat{\alpha} v)=\widehat{g}(u-\widehat{\alpha} v), \quad u, v \in \mathbf{Z}\left(p^{\infty}\right) .
$$

It follows from this that $\widehat{g}(2 \widehat{\alpha} y)=1$ for $y \in \mathbf{Z}\left(p^{\infty}\right)$, and hence $\widehat{g}(y)=1$ for $y \in \mathbf{Z}\left(p^{\infty}\right)$, because $2 \widehat{\alpha}$ is an epimorphism. We proved that $\mu_{1}$, and $\mu_{2}$ are degenerate distributions.

The similar reasoning show that if there exists a sequence of elements $z_{n} \in \mathbf{Z}\left(p^{\infty}\right)$ such that:

(a) the order of element $z_{n}$ is equal to $p^{j_{n}}, j_{n} \rightarrow \infty$;

(b) $\widehat{f}\left(z_{n}\right) \neq 0$,

then $\mu_{1}$, and $\mu_{2}$ are also degenerate distributions.

From what has been said it follows that if $\mu_{1}$, and $\mu_{2}$ are nondegenerate distributions, then there exists $n$ such that $\widehat{f}(y)=\widehat{g}(y)=0$ for $y \notin \mathbf{Z}\left(p^{n}\right)$. Proposition 1 in case 1 follows directly from this.

2. $p>2, k=0, c_{0}=p-1$. Since $p>2$, we have $\widehat{\beta} \in \operatorname{Aut}\left(\mathbf{Z}\left(p^{\infty}\right)\right)$. We find from equation (18) that

$$
\widehat{g}(y)=\widehat{f}\left(2 \widehat{\beta}^{-1} y\right) \widehat{g}\left(\widehat{\gamma} \widehat{\beta}^{-1} y\right), \quad y \in \mathbf{Z}\left(p^{\infty}\right) .
$$

Since $0 \leq \widehat{g}(y) \leq 1$, we find from (26) that

$$
\widehat{g}(y) \leq \widehat{f}\left(2 \widehat{\beta}^{-1} y\right), \quad y \in \mathbf{Z}\left(p^{\infty}\right) .
$$

Reasoning similarly we get from (19) that

$$
\widehat{f}(y)=\widehat{f}\left(\widehat{\gamma} \widehat{\beta}^{-1} y\right) g\left(2 \widehat{\alpha} \widehat{\beta}^{-1} y\right), \quad y \in \mathbf{Z}\left(p^{\infty}\right) .
$$

Taking into account that $0 \leq \widehat{f}(y) \leq 1$, this implies that

$$
\widehat{f}(y) \leq \widehat{g}\left(2 \widehat{\alpha} \widehat{\beta}^{-1} y\right), \quad y \in \mathbf{Z}\left(p^{\infty}\right) .
$$

Inequalities (27) and (29) imply the inequalities

$$
\widehat{g}(y) \leq \widehat{f}\left(2 \widehat{\beta}^{-1} y\right) \leq \widehat{g}\left(4 \widehat{\alpha} \widehat{\beta}^{-2} y\right), \quad \widehat{f}(y) \leq \widehat{g}\left(2 \widehat{\alpha} \widehat{\beta}^{-1} y\right) \leq \widehat{f}\left(4 \widehat{\alpha} \widehat{\beta}^{-2} y\right), \quad y \in \mathbf{Z}\left(p^{\infty}\right)
$$

Reasoning as in the proof of case 1, we find from (30) that

$$
\widehat{g}(y)=\widehat{g}\left(4 \widehat{\alpha} \widehat{\beta}^{-2} y\right), \quad \widehat{f}(y)=\widehat{f}\left(4 \widehat{\alpha} \widehat{\beta}^{-2} y\right), \quad y \in \mathbf{Z}\left(p^{\infty}\right) .
$$

We find from (30) and (31) that

$$
\widehat{g}(y)=\widehat{f}\left(2 \widehat{\beta}^{-1} y\right), \quad \widehat{f}(y)=\widehat{g}\left(2 \widehat{\alpha} \widehat{\beta}^{-1} y\right), \quad y \in \mathbf{Z}\left(p^{\infty}\right) .
$$


It follows from (32) (28) and (26) that if $\widehat{g}\left(y_{0}\right) \neq 0$ for some $y_{0} \in \mathbf{Z}\left(p^{\infty}\right)$, then $\widehat{g}\left(\widehat{\gamma} \widehat{\beta}^{-1} y_{0}\right)=1$, and if $\widehat{f}\left(y_{0}\right) \neq 0$, then $\widehat{f}\left(\widehat{\gamma} \widehat{\beta}^{-1} y_{0}\right)=1$. We complete the proof as in case 1 .

3. $p=2, k=0, c_{0}=c_{1}=1$. Put $\beta=2 \beta_{1}, \gamma=2 \gamma_{1}$. Then $\widehat{\beta}_{1} \in \operatorname{Aut}\left(\mathbf{Z}\left(2^{\infty}\right)\right)$, and $\widehat{\gamma}_{1}$ is an epimorphism. It follows from (18) that

$$
\widehat{f}(y) \widehat{g}\left(\widehat{\gamma}_{1} y\right)=\widehat{g}\left(\widehat{\beta}_{1} y\right), \quad y \in \mathbf{Z}\left(2^{\infty}\right) .
$$

Similarly, we find from (19) that

$$
\widehat{f}\left(\widehat{\gamma}_{1} y\right) \widehat{g}(\widehat{\alpha} y)=\widehat{f}\left(\widehat{\beta}_{1} y\right), \quad y \in \mathbf{Z}\left(2^{\infty}\right) .
$$

It follows from (33) and (34) that

$$
\widehat{g}(y)=\widehat{f}\left(\widehat{\beta}_{1}^{-1} y\right) \widehat{g}\left(\widehat{\gamma}_{1} \widehat{\beta}_{1}^{-1} y\right), \quad \widehat{f}(y)=\widehat{f}\left(\widehat{\gamma}_{1} \widehat{\beta}_{1}^{-1} y\right) \widehat{g}\left(\widehat{\alpha} \widehat{\beta}_{1}^{-1} y\right), \quad y \in \mathbf{Z}\left(2^{\infty}\right) .
$$

Hence,

$$
\widehat{g}(y) \leq \widehat{f}\left(\widehat{\beta}_{1}^{-1} y\right), \quad \widehat{f}(y) \leq \widehat{g}\left(\widehat{\alpha} \widehat{\beta}_{1}^{-1} y\right)
$$

This implies that

$$
\widehat{g}(y) \leq \widehat{f}\left(\widehat{\beta}_{1}^{-1} y\right) \leq \widehat{g}\left(\widehat{\alpha} \beta_{1}^{-2} y\right), \quad \widehat{f}(y) \leq \widehat{g}\left(\widehat{\alpha} \widehat{\beta}_{1}^{-1} y\right) \leq \widehat{f}\left(\widehat{\alpha} \widehat{\beta}_{1}^{-2} y\right)
$$

We complete the proof as in case 2. Proposition 1 is proved completely.

Proof of statement $1($ iii $)$. Observe that in case $1($ iii $) \alpha \neq-I$. Reasoning as in the proof of statements $1(i)$ and $1(i i)$ and retaining the same notation we arrive at equation (14). Put

$$
\begin{array}{ll}
E_{\widehat{f}}=\left\{y \in \mathbf{Z}\left(p^{\infty}\right): \widehat{f}(y) \neq 0\right\}, & E_{\widehat{g}}=\left\{y \in \mathbf{Z}\left(p^{\infty}\right): \widehat{g}(y) \neq 0\right\}, \\
B_{\widehat{f}}=\left\{y \in \mathbf{Z}\left(p^{\infty}\right): \widehat{f}(y)=1\right\}, & B_{\widehat{g}}=\left\{y \in \mathbf{Z}\left(p^{\infty}\right): \widehat{g}(y)=1\right\} .
\end{array}
$$

Since $p>2$ and $k=1$, we have $2 \widehat{\gamma}^{-1} \in \operatorname{Aut}\left(\mathbf{Z}\left(p^{\infty}\right)\right)$. Moreover, since $k=1$, obviously, (20), (21), (23) and (24) hold. Taking this into account, we find from (20) and (23) that $E_{\widehat{g}} \subset B_{\widehat{f}}$. Analogously, we find from (21) and (24) that $p E_{\widehat{f}} \subset B_{\widehat{g}}$. If $\mu_{2}$ is a nondegenerate distribution, then $B_{\widehat{g}}$ is a proper subgroup of $\mathbf{Z}\left(p^{\infty}\right)$, and hence $B_{\widehat{g}}=\mathbf{Z}\left(p^{n}\right)$ for some $n$. Since $B_{\widehat{g}} \subset E_{\widehat{g}} \subset B_{\widehat{f}}$, it follows from (15) that $B_{\widehat{g}}=\{0\}$. If $B_{\widehat{f}}=\{0\}$, then $E_{\widehat{g}} \subset B_{\widehat{f}}=\{0\}$, and hence $\mu_{2}$ is an idempotent distribution. If $B_{\widehat{f}} \neq\{0\}$, then $p B_{\widehat{f}} \subset p E_{\widehat{f}} \subset B_{\widehat{g}}=\{0\}$. This implies that $B_{\widehat{f}}=E_{\widehat{f}}=\mathbf{Z}(p)$, and hence $\mu_{1}$ is an idempotent distribution.

\section{Proof of statements $2(i)-2(i v)$}

Taking into account that the conditional distribution of the linear form $L_{2}$ given $L_{1}$ is symmetric if and only if the conditional distribution of the linear form $\alpha^{-1} L_{2}$ given $L_{1}$ is symmetric, we may assume without loss of generality that $k \geq 0$, and hence the restriction of $\alpha \in \operatorname{Aut}\left(\Omega_{p}\right)$ to the subgroup $\Delta_{p}$ is a continuous endomorphism of $\Delta_{p}$. We retain the notation $\alpha$ for this restriction. We will construct in cases $2(i)-2(i v)$ independent random variables $\xi_{1}$ and $\xi_{2}$ with values in $\Delta_{p}$ and distributions $\mu_{1}$ and $\mu_{2}$ such that the conditional distribution of the linear form $L_{2}=\xi_{1}+\alpha \xi_{2}$ given $L_{1}=\xi_{1}+\xi_{2}$ is symmetric, whereas $\mu_{1}, \mu_{2} \notin I\left(\Delta_{p}\right)$. Considering $\xi_{j}$ as independent random variables with values in $\Omega_{p}$, we prove statements $2(i)-2(i v)$. Taking into account Lemma 1 it suffices to construct non-idempotent distributions $\mu_{j}$ on the group $\Delta_{p}$ such that their characteristic functions satisfy equation (5). We note that the 
groups $\mathbf{Z}\left(p^{\infty}\right)$ and $\Delta_{p}$ are the character groups of one another, and the value of a character $y=\frac{l}{p^{n}} \in \mathbf{Z}\left(p^{\infty}\right)$ at an element $x=\left(x_{0}, x_{1}, \ldots, x_{n}, \ldots\right) \in \Delta_{p}$ is defined by the formula

$$
(x, y)=\exp \left\{\left(x_{0}+x_{1} p+\ldots+x_{n-1} p^{n-1}\right) \frac{2 \pi i l}{p^{n}}\right\} .
$$

Moreover, any topological automorphism of the group $\Delta_{p}$ is the multiplication by an element of $\Delta_{p}^{0}$. For $c=\left(c_{0}, c_{1}, \ldots, c_{n}, \ldots\right) \in \Delta_{p}^{0}$, the restriction of the automorphism $\widetilde{c} \in \operatorname{Aut}\left(\mathbf{Z}\left(p^{\infty}\right)\right)$ to the subgroup $\mathbf{Z}\left(p^{n}\right) \subset \mathbf{Z}\left(p^{\infty}\right)$ is of the form $\widetilde{c} y=s_{n} y, y \in \mathbf{Z}\left(p^{n}\right)$, where $s_{n}=c_{0}+c_{1} p+$ $c_{2} p^{2}+\ldots+c_{n-1} p^{n-1}$. Observe also that $\widetilde{\alpha}=p^{k} \widetilde{c}$, and

$$
A\left(\mathbf{Z}\left(p^{\infty}\right), p^{l} \Delta_{p}\right)=\mathbf{Z}\left(p^{l}\right) .
$$

Put $f(y)=\widehat{\mu}_{1}(y), g(y)=\widehat{\mu}_{2}(y)$. In these notation equation (5) takes the form

$$
f(u+v) g(u+\widetilde{\alpha} v)=f(u-v) g(u-\widetilde{\alpha} v), \quad u, v \in \mathbf{Z}\left(p^{\infty}\right) .
$$

Statement 2(i). Consider on the group $\Delta_{p}$ the distribution $\mu=a m_{\Delta_{p}}+(1-a) m_{p \Delta_{p}}$, where $0<a<1$. It follows from (11) and (35) that the characteristic function $\widehat{\mu}(y)$ is of the form

$$
\widehat{\mu}(y)= \begin{cases}1, & y=0 \\ 1-a, & y \in \mathbf{Z}(p) ; \\ 0, & y \notin \mathbf{Z}(p) .\end{cases}
$$

Let us check that the characteristic functions $f(y)=g(y)=\widehat{\mu}(y)$ satisfy equation (36). Consider 3 cases:

1. $u, v \in \mathbf{Z}(p)$. Since $c_{0}=p-1$, we have $\widetilde{\alpha} y=-y$ for $y \in \mathbf{Z}(p)$, and the restriction of equation (36) to the subgroup $\mathbf{Z}(p)$ takes the form

$$
f(u+v) g(u-v)=f(u-v) g(u+v), \quad u, v \in \mathbf{Z}(p) .
$$

Since $f(y)=g(y)$, (38) holds.

2. Either $u \in \mathbf{Z}(p), v \notin \mathbf{Z}(p)$, or $u \notin \mathbf{Z}(p), v \in \mathbf{Z}(p)$. Then $u \pm v \notin \mathbf{Z}(p)$. This implies that $f(u \pm v)=0$, and hence both sides of equation (36) are equal to zero.

3. $u, v \notin \mathbf{Z}(p)$. If $u+v, u+\widetilde{\alpha} v \in \mathbf{Z}(p)$, then

$$
(I-\widetilde{\alpha}) v \in \mathbf{Z}(p) .
$$

Since $p>2, k=0$ and $c_{0}=p-1$, we have $I-\alpha \in \operatorname{Aut}\left(\Delta_{p}\right)$, and hence $I-\widetilde{\alpha} \in \operatorname{Aut}\left(\mathbf{Z}\left(p^{\infty}\right)\right)$. Then (39) implies that $v \in \mathbf{Z}(p)$, contrary to the assumption. Thus, either $u+v \notin \mathbf{Z}(p)$ or $u+\widetilde{\alpha} v \notin \mathbf{Z}(p)$, and the left-hand side of equation (36) is equal to zero. Similarly we check that the right-hand side of equation (36) is also equal to zero. Thus, (36) holds.

Statement 2(ii). Consider on the group $\Delta_{2}$ the distribution $\mu=a m_{\Delta_{2}}+(1-a) m_{2 \Delta_{2}}$, where $0<a<1$. Then the characteristic function $\widehat{\mu}(y)$ is represented by formula (37) for $p=2$. Let us check that the characteristic functions $f(y)=g(y)=\widehat{\mu}(y)$ satisfy equation (36). Since $k=0$ and $c_{0}=c_{1}=1$, the restriction of the automorphism $\widetilde{\alpha} \in \operatorname{Aut}\left(\mathbf{Z}\left(2^{\infty}\right)\right)$ to the subgroup $\mathbf{Z}\left(2^{n}\right) \subset \mathbf{Z}\left(2^{\infty}\right)$ is of the form $\widetilde{\alpha} y=m y, y \in \mathbf{Z}\left(2^{n}\right)$, where $m=1+2+c_{2} 2^{2}+$ $\ldots+c_{n-1} 2^{n-1}=4 l-1$. Consider 3 cases: 1 . $u, v \in \mathbf{Z}(2) ; 2$. either $u \in \mathbf{Z}(2), v \notin \mathbf{Z}(2)$ or $u \notin \mathbf{Z}(2), v \in \mathbf{Z}(2) ; 3 . u, v \notin \mathbf{Z}(2)$. In cases 1 and 2 the reasoning is the same as in case $2(i)$.

Consider case 3, i.e. assume that $u, v \notin \mathbf{Z}(2)$, and prove first that if $u+v, u+\widetilde{\alpha} v \in \mathbf{Z}(2)$, then $u-v, u-\widetilde{\alpha} v \in \mathbf{Z}(2)$, and (36) holds. To prove this note that the inclusions $u+v, u+\widetilde{\alpha} v \in$ $\mathbf{Z}(2)$ are possible only in the following cases. 
(a) $u+v=0$ and $u+\widetilde{\alpha} v=0$. This implies that $(\widetilde{\alpha}-I) v=(m-1) v=2(2 l-1) v=0$. Hence, $v \in \mathbf{Z}(2)$, but this contradicts to the assumption.

(b) $u+v=\frac{1}{2}$ and $u+\widetilde{\alpha} v=\frac{1}{2}$. The reasoning is the same as in case $(a)$.

(c) $u+v=0$ and $u+\widetilde{\alpha} v=\frac{1}{2}$. This implies that $(\widetilde{\alpha}-I) v=(m-1) v=2(2 l-1) v=\frac{1}{2}$. It follows from this that either $v=\frac{1}{4}, u=\frac{3}{4}$, or $v=\frac{3}{4}, u=\frac{1}{4}$. In both cases $u-v=\frac{1}{2}$, $u-\widetilde{\alpha} v=u-m v=0$, and hence (36) holds.

(d) $u+v=\frac{1}{2}, u+\widetilde{\alpha} v=0$. The reasoning is the same as in case $(c)$.

Reasoning similarly we verify that if $u-v, u-\widetilde{\alpha} v \in \mathbf{Z}(2)$, then $u+v, u+\widetilde{\alpha} v \in \mathbf{Z}(2)$, and (36) holds.

Statement 2(iii). Consider on the group $\Delta_{2}$ the distributions $\mu_{1}=a m_{2 \Delta_{2}}+(1-a) m_{4 \Delta_{2}}$ and $\mu_{2}=a m_{\Delta_{2}}+(1-a) m_{2 \Delta_{2}}$, where $0<a<1$. It follows from (11) and (35) that that the characteristic function $\widehat{\mu}_{1}(y)$ is of the form

$$
\widehat{\mu}_{1}(y)= \begin{cases}1, & y \in \mathbf{Z}(2) ; \\ 1-a, & y \in \mathbf{Z}(4) ; \\ 0, & y \notin \mathbf{Z}(4) .\end{cases}
$$

Moreover, the characteristic function $\widehat{\mu}_{2}(y)$ is represented by formula (37) for $p=2$. Let us check that the characteristic functions $f(y)=\widehat{\mu}_{1}(y)$ and $g(y)=\widehat{\mu}_{2}(y)$ satisfy equation (36). Consider 3 cases:

1. $u, v \in \mathbf{Z}(4)$. Obviously, we can assume that $u \neq 0, v \neq 0$. Since either $c_{0}=1, c_{1}=0$ or $c_{0}=c_{1}=1$, the restriction of the automorphism $\widetilde{c}$ to the subgroup $\mathbf{Z}(4)$ is of the form: either $\widetilde{y}=y$ or $\widetilde{y}=-y, y \in \mathbf{Z}(4)$. Thus, the restriction of equation (36) to the subgroup $\mathbf{Z}(4)$ either takes the form

$$
f(u+v) g(u+2 v)=f(u-v) g(u-2 v), \quad u, v \in \mathbf{Z}(4),
$$

or

$$
f(u+v) g(u-2 v)=f(u-v) g(u+2 v), \quad u, v \in \mathbf{Z}(4) .
$$

Consider equation (40). Equation (41) can be considered analogously.

(a) $u=v=\frac{1}{2}$. Then $u \pm v=0,2 v=0$. Hence, $f(u \pm v)=1, g(u \pm 2 v)=g(u)$ and (40) holds.

(b) $u=\frac{1}{2}, v \in\left\{\frac{1}{4}, \frac{3}{4}\right\}$. Then $f(u \pm v)=f(v)$. Since $u \pm 2 v=0$, we have $g(u \pm 2 v)=1$, and (40) holds.

(c) $u \in\left\{\frac{1}{4}, \frac{3}{4}\right\}, v=\frac{1}{2}$. Then $f(u \pm v)=f(u)$. Since $2 v=0$, we have $g(u \pm 2 v)=g(u)$, and (40) holds.

(d) $u, v \in\left\{\frac{1}{4}, \frac{3}{4}\right\}$. This implies that $u \pm 2 v \notin \mathbf{Z}(2)$. Hence $g(u \pm 2 v)=0$, and both sides of equation (40) are equal to zero.

2. Either $u \in \mathbf{Z}(4), v \notin \mathbf{Z}(4)$ or $u \notin \mathbf{Z}(4), v \in \mathbf{Z}(4)$. Then $u \pm v \notin \mathbf{Z}(4)$. Hence $f(u \pm v)=0$ and both sides of equation (40) are equal to zero.

3. $u, v \notin \mathbf{Z}(4)$. If $u+v \in \mathbf{Z}(4)$ and $u+\widetilde{\alpha} v \in \mathbf{Z}(2)$, then

$$
(I-\widetilde{\alpha}) v \in \mathbf{Z}(4) .
$$

Since $k=1$, we have $I-\alpha \in \operatorname{Aut}\left(\Delta_{2}\right)$, and hence $I-\widetilde{\alpha} \in \operatorname{Aut}\left(\mathbf{Z}\left(2^{\infty}\right)\right)$. Then (42) implies that $v \in \mathbf{Z}(4)$, contrary to the assumption. Hence, either $u+v \notin \mathbf{Z}(4)$ or $u+\widetilde{\alpha} v \notin \mathbf{Z}(2)$. This implies that the left-hand side of equation (36) is equal to zero. Reasoning analogously, we verify that the right-hand side of equation (36) is also equal to zero. Thus, (36) holds. 
Statement $2(i v)$. Consider on the group $\Delta_{p}$ the distributions $\mu_{1}=a m_{p^{k-1} \Delta_{p}}+(1-$ a) $m_{p^{k} \Delta_{p}}$ and $\mu_{2}=a m_{\Delta_{p}}+(1-a) m_{p^{k-1} \Delta_{p}}$, where $0<a<1$. It follows from (11) and (35) that the characteristic functions $\widehat{\mu}_{1}(y)$ and $\widehat{\mu}_{2}(y)$ are of the form

$$
\widehat{\mu}_{1}(y)=\left\{\begin{array}{ll}
1, & y \in \mathbf{Z}\left(p^{k-1}\right) ; \\
1-a, & y \in \mathbf{Z}\left(p^{k}\right) \backslash \mathbf{Z}\left(p^{k-1}\right) ; \\
0, & y \notin \mathbf{Z}\left(p^{k}\right) .
\end{array} \quad \widehat{\mu}_{2}(y)= \begin{cases}1, & y=0 ; \\
1-a, & y \in \mathbf{Z}\left(p^{k-1}\right) \backslash\{0\} ; \\
0, & y \notin \mathbf{Z}\left(p^{k-1}\right) .\end{cases}\right.
$$

Let us check that the characteristic functions $f(y)=\widehat{\mu}_{1}(y)$ and $g(y)=\widehat{\mu}_{2}(y)$ satisfy equation (36). Consider 3 cases:

1. $u, v \in \mathbf{Z}\left(p^{k}\right)$. Since $\alpha=p^{k} c$, we have $\widetilde{\alpha} v=0$, and hence the restriction of equation (36) to the subgroup $\mathbf{Z}\left(p^{k}\right)$ takes the form

$$
f(u+v) g(u)=f(u-v) g(u), \quad u, v \in \mathbf{Z}\left(p^{k}\right) .
$$

(a) $u \in \mathbf{Z}\left(p^{k-1}\right)$. Then $f(u \pm v)=f(v)$, and equation (44) holds.

(b) $u \in \mathbf{Z}\left(p^{k}\right) \backslash \mathbf{Z}\left(p^{k-1}\right)$. Then $g(u)=0$, and both sides of equation (44) are equal to zero.

2. Either $u \in \mathbf{Z}\left(p^{k}\right), v \notin \mathbf{Z}\left(p^{k}\right)$ or $u \notin \mathbf{Z}\left(p^{k}\right), v \in \mathbf{Z}\left(p^{k}\right)$. Then $u \pm v \notin \mathbf{Z}\left(p^{k}\right)$. This implies that $f(u \pm v)=0$, and both sides of equation (36) are equal to zero.

3. $u, v \notin \mathbf{Z}\left(p^{k}\right)$. If $u+v \in \mathbf{Z}\left(p^{k}\right), u+\widetilde{\alpha} v \in \mathbf{Z}\left(p^{k-1}\right)$, then

$$
(I-\widetilde{\alpha}) v \in \mathbf{Z}\left(p^{k}\right) .
$$

Since $k \geq 1$, we have $I-\alpha \in \operatorname{Aut}\left(\Delta_{p}\right)$, and hence $I-\widetilde{\alpha} \in \operatorname{Aut}\left(\mathbf{Z}\left(p^{\infty}\right)\right)$. Then (45) implies that $v \in \mathbf{Z}\left(p^{k}\right)$, contrary to the assumption. Hence, either $u+v \notin \mathbf{Z}\left(p^{k}\right)$ or $u+\widetilde{\alpha} v \notin \mathbf{Z}\left(p^{k-1}\right)$. This implies that the left-hand side of equation (36) is equal to zero. Reasoning analogously, we verify that the right-hand side of equation (36) is also equal to zero. Thus, (36) holds. Theorem 1 is proved.

Let $X=\Delta_{p}$. We remind that each automorphism $\alpha \in \operatorname{Aut}\left(\Delta_{p}\right)$ is the multiplication by an element $c_{\alpha} \in \Delta_{p}^{0}$. Let $\xi_{1}$ and $\xi_{2}$ be independent random variables with values in the group $\Delta_{p}$. Then we can consider $\xi_{j}$ as independent random variables with values in the group $\Omega_{p}$. Moreover, it is obvious that the multiplication by an element $c \in \Delta_{p}^{0}$ is a topological isomorphism of the group $\Omega_{p}$. This implies that statements $1(i)$ and $1(i i)$ in Theorem 1 are also valid for the group $\Delta_{p}$. Taking into account that in the proof of statements $2(i)$ and $2(i i)$ in Theorem 1 the corresponding independent random variables take values in the subgroup $\Delta_{p} \subset \Omega_{p}$, we conclude that the following theorem holds.

Theorem 2. Let $X=\Delta_{p}$. Let $\alpha=c=\left(c_{0}, c_{1}, \ldots, c_{n}, \ldots\right) \in \Delta_{p}^{0}$ be an arbitrary topological automorphism of the group $X$. Then the following statements hold.

1. Let $\xi_{1}$ and $\xi_{2}$ be independent random variables with values in $X$ and distributions $\mu_{1}$ and $\mu_{2}$. Assume that the conditional distribution of the linear form $L_{2}=\xi_{1}+\alpha \xi_{2}$ given $L_{1}=\xi_{1}+\xi_{2}$ is symmetric. Then

$1(i)$ If $p>2$ and $c_{0} \neq p-1$, then $\mu_{j}=m_{K} * E_{x_{j}}$, where $K$ is a compact subgroup of $X$ and $x_{j} \in X$. Moreover, if $c_{0}=1$, then $\mu_{1}$ and $\mu_{2}$ are degenerate distributions.

1 (ii) If $p=2, c_{0}=1$ and $c_{1}=0$, then $\mu_{1}$ and $\mu_{2}$ are degenerate distributions.

2. If one of the following conditions holds:

$2(i) p>2, c_{0}=p-1$;

2 (ii) $p=2, c_{0}=c_{1}=1$,

then there exist independent random variables $\xi_{1}$ and $\xi_{2}$ with values in $X$ and distributions $\mu_{1}$ and $\mu_{2}$ such that the conditional distribution of the linear form $L_{2}=\xi_{1}+\alpha \xi_{2}$ given $L_{1}=\xi_{1}+\xi_{2}$ is symmetric whereas $\mu_{1}, \mu_{2} \notin I(X)$. 
Remark 1. Assume that in Theorem $1 p>2, k=0, c_{0} \neq 1$ and $c_{0} \neq p-1$. This implies in particular, that $I-\alpha \in \operatorname{Aut}\left(\Delta_{p}\right)$, and hence

$$
I-\widetilde{\alpha} \in \operatorname{Aut}\left(\mathbf{Z}\left(p^{\infty}\right)\right) .
$$

Let $\xi_{1}$ and $\xi_{2}$ be independent identically distributed random variables with values in $\Delta_{p}$ and distribution $m_{\Delta_{p}}$. We check that the conditional distribution of the linear form $L_{2}=\xi_{1}+\alpha \xi_{2}$ given $L_{1}=\xi_{1}+\xi_{2}$ is symmetric. By Lemma 1 , it suffices to verify that the characteristic functions $f(y)=g(y)=\widehat{m}_{\Delta_{p}}(y)$ satisfy equation (36). It follows from (1) that

$$
\widehat{m}_{\Delta_{p}}(y)= \begin{cases}1, & y=0 \\ 0, & y \neq 0\end{cases}
$$

Obviously, it suffices to check that equation (36) holds when $u \neq 0, v \neq 0$. Thus, assume that $u \neq 0, v \neq 0$. If $u+v=0$ and $u+\widetilde{\alpha} v=0$, then $(I-\widetilde{\alpha}) v=0$. Taking into account (46), this implies that $v=0$, contrary to the assumption. Thus, either $u+v \neq 0$ or $u+\widetilde{\alpha} v \neq 0$, and hence the left-hand side of equation (36) is equal to zero. Reasoning analogously we show that the right-hand side of equation (36) is also equal to zero. So, equation (36) holds. This example shows that statement $1(i)$ in Theorem 1 can not be strengthened to the statement that both $\mu_{1}$ and $\mu_{2}$ are degenerate distributions.

Assume that conditions 1 ( iii) of Theorem 1 hold, i.e. $p>2$ and $|k|=1$. We can assume without loss of generality that $k=1$. Let $\xi_{1}$ and $\xi_{2}$ be independent random variables with values in the group $\Delta_{p}$ and distributions $\mu_{1}=a m_{\Delta_{p}}+(1-a) m_{p \Delta_{p}}$, where $0<a<1$, and $\mu_{2}=m_{\Delta_{p}}$. Then the characteristic functions $f(y)=\widehat{\mu}_{1}(y)$ and $g(y)=\widehat{\mu}_{2}(y)$ are defined by formulas (43) for $k=1$. Obviously, the proof that the characteristic functions $f(y)$ and $g(y)$ satisfy equation (36), given in the proof of statement $2(i v)$, remains true for $k=1$ too. Thus, statement 1 (iii) can not be strengthened to the statement that both distributions $\mu_{1}, \mu_{2} \in I(X)$.

Remark 2. Compare Theorem 1 with Heyde's characterization theorem for two independent random variables on the real line $X=\mathbf{R}$. It is easy to see that this theorem can be formulated in the following way. Let $\xi_{1}$ and $\xi_{2}$ be independent random variables with values in the group $X=\mathbf{R}$ and distributions $\mu_{1}$ and $\mu_{2}$. Let $\alpha \neq-I$. If the conditional distribution of the linear form $L_{2}=\xi_{1}+\alpha \xi_{2}$ given $L_{1}=\xi_{1}+\xi_{2}$ is symmetric, then $\mu_{j}$ are Gaussian. Thus, on the real line the only condition $\alpha \neq-I$, obviously, necessary, is sufficient for characterization of the Gaussian distribution.

It follows from Theorem 1 that on the group of $p$-adic numbers $X=\Omega_{p}$ the state of affairs is more complicated. On the one hand, much more severe constrains for $\alpha$ (conditions $1(i)$ for $p>2$ and $1(i i)$ for $p=2$ ) are necessary and sufficient for characterization of the idempotent distribution. On the other hand, there exist $\alpha$ (satisfying conditions $1(i i i)$ ) such that the symmetry of the conditional distribution of the linear form $L_{2}=\xi_{1}+\alpha \xi_{2}$ given $L_{1}=\xi_{1}+\xi_{2}$ implies that the only one of the distributions $\mu_{j}$ is idempotent. This effect is absent on the real line.

\section{References}

1. G.M. Feldman, On the Heyde theorem for finite Abelian groups, J. Theoretical Probab. 17, 929-941 (2004).

2. G.M. Feldman, On the Heyde theorem for discrete Abelian groups, Studia Math. 177, 67-79 (2006). 
3. G.M. Feldman, On a characterization theorem for locally compact abelian groups, Probab. Theory Relat. Fields 133, 345-357 (2005).

4. G.M. Feldman, Functional equations and characterization problems on locally compact Abelian groups, EMS Tracts in Mathematics 5, European Mathematical Society, Zurich, 2008.

5. G.M. Feldman, The Heyde theorem for locally compact Abelian groups, J. Funct. Anal. 258, No 12, 3977-3987 (2010).

6. G.M. Feldman, On a characterization of convolutions of Gaussian and Haar distributions, Math. Nachr. 286, 340-348 (2013).

7. E. Hewitt, and K.A. Ross, Abstract Harmonic Analysis, vol. 1, Springer-Verlag, Berlin, Gottingen, Heildelberg, 1963.

8. C.C. Heyde, Characterization of the normal low by the symmetry of a certain conditional distribution, Sankhya 32, Ser. A. 115-118 (1970).

9. A. M. Kagan, Yu. V. Linnik, C.R. Rao, Characterization problems in mathematical statistics, Wiley Series in Probability and Mathematical Statistics, John Wiley \& Sons, New York-London-Sydney, 1973.

10. M. V. Myronyuk, Heyde's characterization theorem for discrete Abelain groups, Journal. of Australian Math. Soc. 88, 93-102 (2010).

11. M. V. Myronyuk, The Heyde theorem on $\boldsymbol{a}$-adic solenoids, Colloquium Mathematiquim 132, 195-210 (2013).

12. M.V. Myronyuk, G.M. Fel'dman, On a characterization theorem on finite abelian groups, Siberian Math. J. 46, 315-324 (2005).

13. K.P. Parthasarathy, Probability measures on metric spaces, New York and London: Academic Press, 1967. 